\title{
A OBSOLESCÊNCIA TECNOLÓGICA E A FRAGILIDADE DOS SUPORTES DE DOCUMENTOS DIGITAIS
}

\section{TECHNOLOGICAL OBSOLESCENCE AND THE FRAGILITY OF DIGITAL DOCUMENT SUPPORTS}

\author{
Mariane Costa Pintoa \\ Thaiane Honda Cotts ${ }^{b}$
}

\begin{abstract}
RESUMO
Introdução: Este trabalho discute a obsolescência tecnológica e fragilidade dos suportes dos documentos arquivísticos digitais e o que isso implica na sua preservação. Objetivo: Elaborou-se uma revisão de literatura para analisar em que grau encontra-se a discussão sobre preservação digital e apresentar propostas para a preservação dos documentos arquivísticos digitais diante da velocidade do avanço tecnológico. Metodologia: Realizou-se um levantamento bibliográfico acerca do tema publicado em livros, teses, sítios da internet e artigos de periódicos, entre outros materiais, principalmente em língua portuguesa. A coleta de dados tem cunho qualitativo. Resultados: Como resultado pode-se considerar que a obsolescência tecnológica é consequência da celeridade das mudanças e avanços na área que atingem não só a internet como os formatos de arquivo, softwares, hardwares e sistemas operacionais. Isso obriga ao profissional de preservação a agir rapidamente a fim de proteger a história da sociedade presente nos documentos e colocada em risco. Conclusões: Conclui-se que é essencial a criação de estratégias para preservação digital, com isso será possível um monitoramento, planejamento e tratamento a longo prazo que minimize as consequências dessa obsolescência a fim de preservar sua originalidade e autenticidade.
\end{abstract}

Descritores: Obsolescência tecnológica. Preservação digital . Documentos arquivísticos Documentos digitais .

\section{INTRODUÇÃO}

O presente trabalho tem como objetivo trazer à tona uma reflexão pertinente à Arquivologia contemporânea, a preservação digital da informação

\footnotetext{
a Doutoranda em Ciência da Informação pela Universidade de Coimbra. E-mail: marianecpinto@hotmail.com.

b Doutoranda em Ciência da Informação pela Universidade de Coimbra. E-mail: thondacotts@gmail.com.
} 
diante das constantes mudanças nas tecnologias e suportes dos documentos.

$O$ inegável avanço tecnológico na sociedade a partir do século $X X$, impulsionou a produção de documentos por meio digital, muito por conta da disseminação dos computadores, instrumento que tornou-se indispensável para a realização de atividades. (SANTOS; FLORES, 2016)

Com isso, veio o desafio de preservar os documentos digitais arquivísticos mantendo sua originalidade, autenticidade e confiabilidade - por conta de seu caráter probatório - e, ao mesmo tempo, enfrentar a fragilidade do documento digital, graças à sua rápida degradação física e obsolescência tecnológica.

A preservação dos documentos digitais requer ações arquivísticas, a serem incorporadas em todo o seu ciclo de vida, antes mesmo de terem sido criados, incluindo as etapas de planejamento e concepção de sistemas eletrônicos, a fim de que não haja perda nem adulteração dos registros. Somente desta forma se garantirá que esses documentos permaneçam disponíveis, recuperáveis e compreensíveis pelo tempo que se fizer necessário. (CONARQ, 2005, p. 2)

O documento arquivístico possui sua especificidade desde o momento de sua criação, uma vez que possui um caráter primeiro administrativo, para depois da avaliação tornar-se cultural. Por conta disso, precisa manter sua integridade, autenticidade, capacidade probatória e manter o seu contexto de produção. Os usuários de arquivo necessitam acessar documentos autênticos e a longo prazo, o que faz com que se discuta a relevância da preservação de documentos em ambiente digital. (SANTOS; FLORES, 2015b)

Isto posto, mostra-se fundamental para profissionais da informação refletir acerca de como lidar com essa explosão informacional e constantes mudanças tecnológicas, tendo em mente o seu principal papel de preservar e dar acesso a informação à toda a sociedade.

\section{METODOLOGIA}

A metodologia utilizada apoia-se no levantamento bibliográficodocumental de materiais publicados sobre assunto e inclui: livros, dissertações, teses, textos da internet, artigos de periódicos, publicações de órgãos oficiais de Arquivologia, principalmente de língua portuguesa. (SANTOS; FLORES, 2015a) 
Constitui-se de uma revisão de literatura, de abordagem qualitativa, que aborda publicações recentes, principalmente dos últimos dez anos, embora resgate também publicações anteriores devido a relevância para o tema.

Como se trata de uma reflexão ainda recente e aberta, o texto tem foco abrangente, partindo do aspecto geral para o específico, abordando estratégias para a resolução do problema.

\section{PRESERVAÇÃO DIGITAL: UMA BREVE INTRODUÇÃO}

A mudança na produção de documentos arquivísticos é uma realidade. Cada vez mais, as organizações públicas e privadas produzem textos, planilhas, bases de dados e arquivos audiovisuais, entre outros formatos, por meio digital. A mudança de paradigma facilita a troca e difusão da informação arquivística e reduz os custos. (CONARQ, 2005; SANTOS; FLORES, 2015a)

A avaliação do prazo necessário de guarda e destinação final nos documentos arquivísticos digitais também é um desafio, pois depende que o sistema de informação que o armazena tenha como requisito a tabela de temporalidade, e ainda sim, dependerá da análise correta do arquivista. (SANTOS; FLORES, 2015c)

Vale destacar que as sete funções arquivísticas citadas por Rousseau e Couture (1998 apud SANTOS; FLORES, 2015c): produção, avaliação, aquisição, conservação, classificação, descrição e difusão são aplicáveis a qualquer documento independente da idade ou formato, entretanto, é inegável a necessidade de adaptações aos documentos digitais. Acerca da preservação destas documentações muitas são as questões que precisam ser respondidas.

The preservation of digital objects involves a variety of challenges, including policy questions, institutional roles and relationships, legal issues, intellectual property rights, and metadata. But behind or perhaps beneath such issues, there are substantial challenges at the empirical level. What does it mean to preserve digital objects? What purposes are served by preserving them? What are the real possibilities for successful preservation? What are the problems encountered in trying to exploit these possibilities? Can we articulate a framework or an overall architecture for digital preservation that allows us to discriminate and select possibilities? (THIBODEAU, [2002], p. 1) 
Pode-se considerar preservação digital como "um conjunto de objetivos e métodos para efetuar a manutenção em longo prazo dos documentos digitais, contemplando os seus respectivos objetos digitais e as suas informações relacionadas". (SANTOS; FLORES, 2015d)

O responsável pela preservação precisa definir o escopo e os objetivos do seu programa de preservação digital, levando em consideração a acessibilidade dos dados no futuro, os usuários desta documentação e a complexidade dos materiais digitais (INTERPARES, [2013]).

A preservação digital exige conhecimento especializado e recursos constantes para incremento tecnológico. Além disso, o investimento em cópias oficiais digitais é mais importante do que em cópias digitalizadas de documentos analógicos, visto que o original analógico terá durabilidade e não dependerá da digitalização, enquanto o digital, uma vez perdido, não haverá outra maneira de acessá-lo (INTERPARES, [2013]).

De acordo com Ferreira (2006), muitas são as tentativas de criar estratégias para a preservação digital, Lee e seus colaboradores, dividiram essas estratégias em três grandes classes: emulação, migração e encapsulamento. Thibodeau considera duas: a centrada no objeto físico/lógico e a centrada no objeto conceitual que são aplicáveis de acordo com o grau de especificidade.

Para a preservação digital de documentos em arquivos são necessárias ações como a preservação tecnológica, a migração, a emulação, o encapsulamento, adoção de padrões e protocolos, adoção de política de gestão documental e tecnológica, controle público de legitimidade, além de uma política pública que inclua pesquisa científica, assim como ações por partes de arquivos, em todos os níveis. Isso dependerá de legislação apropriada (BAGGIO; FLORES, 2012, p. 62).

Contudo, é importante que se perceba que qualquer estratégia de preservação digital depende da possibilidade de aplicá-la. Não existe uma aplicação que possa ser usada em todos em casos. Nem sempre a preservação de um objeto digital inclui a manutenção de todos os seus componentes. As estratégias variam de acordo com a aplicabilidade em softwares, hardwares ou dados individuais (THIBODEAU, [2002]). 


\section{A OBSOLESCÊNCIA TECNOLÓGICA E A FRAGILIDADE DOS SUPORTES}

A obsolescência tecnológica é um dos maiores desafios da preservação digital, pois, diferente do documento analógico, o digital depende exclusivamente de um ambiente tecnológico complexo composto de fatores físicos (hardwares) e lógicos (softwares). As rápidas mudanças nos componentes tecnológicos deixam os documentos digitais mais suscetíveis à degradação física (FERREIRA, C., 2011).

A obsolescência tecnológica não se manifesta somente ao nível
dos suportes físicos. No domínio digital, todo o tipo de material
tem obrigatoriamente de respeitar as regras de um determinado
formato. Isto permite que as aplicações de software sejam
capazes de abrir e interpretar adequadamente a informação
armazenada. À medida que o software vai evoluindo, também os
formatos por ele produzidos vão sofrendo alterações
(FERREIRA, M., 2006, p. 19).

Dentre os maiores esforços por parte do preservador está a manutenção do acesso ao documento digital, independente do contexto tecnológico que esteja inserido. Não é viável que a organização mantenha a informação multiplicada em todos os suportes tecnológicos no qual passará ao longo dos anos, é interessante que o preservador sempre transfira a informação para o suporte mais atual ou crie emuladores que "leiam" o ambiente original do documento (FERREIRA, C., 2011). Esta opção deve ser utilizada somente quando não for possível converter o documento a softwares independentes que permitam migração futura (BAGGIO; FLORES, 2012).

Sabe-se que o documento digital possui uma fragilidade muito maior do que o analógico, uma vez que este tem a possibilidade de resistir ao tempo, especialmente se em boas condições de temperatura e encapsulamento, já aquele depende de diversas decisões - que perpassam as situações já citadas de degradação física, obsolescência tecnológica, transferência de dados - e de conhecimentos de preservação específicos.

Enquanto se pensa em uma durabilidade do papel de décadas, séculos, milênios, no documento digital se pensa em anos, e até meses. Esta rapidez na obsolescência tecnológica, e que pode trazer como consequência a falta de 
acesso a um documento probatório, implica em uma necessidade de investimento em preservação muito mais complexa, especialmente por conta do volume de produção de documentos digitais nas organizações e na responsabilidade em mantê-los acessíveis em longo prazo (SANTOS; FLORES, 2015c).

A capacidade de reprodução da informação digital torna-a, teoricamente, invulnerável às ações do tempo, entretanto, o suporte não possui tais características (Rothenberg, 1999). Além disso, o acesso aos documentos digitais depende de um software interpretador, o qual deve processar corretamente as sequências de bits, e ainda ser capaz de interoperar com outros softwares. Neste caso, não se recomenda o uso de softwares incompatíveis com versões anteriores ou com versões posteriores, pois estes softwares dificultam o acesso aos documentos digitais em longo prazo (INTERPARES 2 PROJECT, 2007b apud SANTOS; FLORES 2017, p. 31).

O alto custo operacional da preservação digital muitas vezes inviabiliza a preservação digital em longo prazo. Com ciclos de mudança de tecnologia acelerados, a mudança de peças e aperfeiçoamento dos profissionais fica cada vez mais caro e complicado de operacionalizar.

Carla Ferreira (2011) destaca que os maiores desafios são: obsolescência do hardware e dos suportes; a obsolescência do software e obsolescência de formatos. A autora afirma que, com a vinda de determinada inovação tecnológica, vem, muitas vezes, a necessidade de troca de periféricos e dispositivos de leitura o que muitas vezes impossibilita o acesso aos documentos digitais da organização.

O software enfrenta a necessidade de manter as atualizações com capacidade de decodificar os dados das versões anteriores, mesmo que as mais antigas, para que o documento digital possa ser acessado. As atualizações de softwares podem vir acompanhadas de uma mudança nos formatos. Com isso, se as mudanças os tornarem incompatíveis ou o formato for descontinuado, os documentos também podem deixar de ser acessados (FERREIRA, C., 2011).

\section{ESTRATÉGIAS PARA PRESERVAÇÃO DIGITAL}

Diversos autores propõem como ações para a preservação digital: migração, emulação, encapsulamento, conservação de hardware e software, 
reprografia, refrescamento, atualização de versões, conversão para formatos concorrentes, normalização, a Pedra de Rosetta Digital e a arqueologia digital (BAGGIO; FLORES, 2012; FERREIRA, 2006; FERREIRA, C., 2011).

\subsection{MigRaÇÃo}

A migração é importante pois realiza a transferência da informação de um formato antigo para o compatível/atual. Este método permite o acesso permanente à informação, pois preocupa-se com o conteúdo intelectual do documento.

Nesta estratégia, a preocupação é manter a integridade dos objetos digitais para assegurar que seja possível recuperá-los. Ao torná-lo compatível com as tecnologias atuais, este método dispensa o uso de outros dispositivos como a emulação (FERREIRA, 2006).

Está voltada para preservação do objeto conceitual no qual o documento digital pode ser acessado independente da forma, entretanto não contempla todos os tipos de objetos digitais. Além disso, alguns objetos correm o risco de não serem transferidos corretamente, devido a alguma incompatibilidade entre $o$ formato de origem e o destinado (FERREIRA, 2006).

\subsection{EMULAÇÃo}

A emulação permite que a execução de um documento digital seja feita em um novo sistema desenvolvido com a habilidade de permitir a sua leitura da mesma forma que ocorria em um software já obsoleto (BAGGIO; FLORES, 2012).

De acordo com Ferreira (2006), este tipo de ação tem foco na preservação do objeto lógico. Tem como principal vantagem, o alto grau de fidelidade às características do documento digital.

São dois os tipos de emuladores, os de hardware, que visa replicar o comportamento de uma plataforma de hardware em diversas aplicativos e sistemas operacionais, e os de sistemas operacionais, no qual se reproduz completamente o sistema operacional a fim de que se possa executar vários 
aplicativos em um único emulador (FERREIRA, 2006).

\subsection{ENCAPSULAMENTO}

O encapsulamento refere-se na manutenção no objeto digital de os recursos necessários para o futuro desenvolvimento de emuladores, conversores e visualizadores. Podem ser metadados, softwares visualizadores ou arquivos específicos que sejam parte do recurso digital (FERREIRA, 2006; BAGGIO; FLORES, 2012).

Este procedimento mantém os originais inalterados até o momento do uso, mas necessita vir acompanhado de todas as instruções para interpretação dos formatos dos documentos e do conteúdo da informação (BAGGIO; FLORES, 2012).

\subsection{Conservação de HaRdWARE E Software}

De acordo com Baggio e Flores (2012) ainda há organizações que optam pela conservação do hardware e do software, mas para a preservação digital é uma decisão muito complexa e cara. A melhor solução seria criar componentes compatíveis.

Seu maior problema está na criação de grandes museus de tecnologia, uma vez que as tecnologias mudam de maneira muito rápida. Trata-se de uma preservação não do objeto lógico, conceitual, mas do objeto físico (FERREIRA, 2006).

\subsection{REPROGRAFIA}

Outra ação implica na digitalização dos documentos "em papel, foto, microfilme, microficha, jaqueta ou cartão janela" (BAGGIO; FLORES, 2012, p. 66). Segundo o Conselho Nacional de Arquivos do Brasil, a digitalização é uma das atividades fundamentais para a difusão e o acesso à documentação arquivística e contribui para sua preservação uma vez que evita o manuseio dos originais (BAGGIO; FLORES, 2012).

A digitalização é um processo que trata da transformação da informação 
analógica em digital (FERREIRA, M., 2006). Passou a ser utilizada a partir dos anos 80 e com o advento da internet, a partir dos anos 90 , tornou-se importante ferramenta de difusão e acesso (FERREIRA, C., 2011).

Apesar de considerada "a mais flexível ferramenta de arquivamento, preservação e acesso a documentos" (p. 66), existem recomendações específicas para captura da imagem em diversos formatos, produção de matrizes e derivadas e boas práticas para armazenamento, preservação e segurança desses representantes digitais (BAGGIO; FLORES, 2012).

\subsection{REFRESCAMENTO}

O refrescamento é a transferência da informação digital de um suporte para outro mais atual antes da sua deterioração. Uma de suas vantagens é a ausência de necessidade de alto investimento em equipamentos e conhecimento técnico específico, à exceção dos casos de informação protegida (BAGGIO; FLORES, 2012).

Pode ser considerado um pré-requisito da estratégia de preservação, uma vez que a frequente verificação dos suportes e o refrescamento periódico são atividades vitais e que devem ser realizadas periodicamente como forma de preservação digital (FERREIRA, 2006).

\subsection{AtUalização das Versões}

A atualização de versões é a estratégia mais amplamente conhecida e usada pelos usuários, consiste basicamente na mesma ação de transferência, mas de um software para outro mais atual. É muito comum que versões mais recentes de software possibilitem a importação dos objetos digitais das versões anteriores (BAGGIO; FLORES, 2012).

Version migration sets up a chain that must be extended over time, because every format will eventually become obsolete. One problem with this approach is that using more recent versions of software, even with the original formats, may present the preserved documents with characteristics they did not, and perhaps could not, have had. For example, any document created with a word processor in the early 1990s, before "WYSIWYG" display was available, would have appeared on screen with a black background and green letters. If one were to 
open such a document with a word processor today, it would look much like a printed page.(THIBODEAU, [2002], p. [21])

\subsection{Conversão Para Formatos Concorrentes}

A conversão para formatos de empresas concorrentes é restrita a alguns objetos, mas pode ser uma boa opção. A qualidade da conversão depende da capacidade de retrocompatibilidade. (BAGGIO e FLORES, 2012)

Esta ação é considerada porque, por mais que quase sempre se possa converter um objeto digital de um formato anterior de software para um mais recente da mesma empresa, algumas vezes eles podem acabar descontinuados e o acesso ao documento fica em risco. Resta à organização tentar a conversão deste objeto para um formato de outra empresa de software. (FERREIRA, 2006)

\subsection{NORMALIZAÇÃo}

Outra estratégia a ser utilizada é a normalização de formatos para aplicação transversal a um número maior de objetos. Seu principal objetivo é reduzir a quantidade de formatos, aumentando seu controle e simplificando 0 processo de preservação. (FERREIRA, M., 2006)

Vejamos um exemplo. Existe um leque variado de opções no que diz respeito a formatos para representação de imagens bidimensionais (e.g. BMP, GIF, JPEG, PNG, TARGA). Se durante o processo de ingestão, todas as imagens digitais forem convertidas para um único formato, futuras intervenções ao nível da sua preservação poderão ser realizadas de forma mais simples e, consequentemente, mais económica. (FERREIRA, M., 2006, p. 39)

A normalização pode acontecer de várias formas, há repositórios que o fazem automaticamente, outros que definem políticas para limitar formatos, mas são os produtores da informação os mais indicados a avaliar a qualidade das conversões. (BAGGIO; FLORES, 2012)

\subsection{Pedra de Rosetta Digital}

A Pedra de Rosetta Digital pode ser utilizada para recuperar informações sobre o formato de uma informação digital, quando estas não estão por completo 
nos objetos digitais.

Faz-se por meio de uma reunião de amostras dessa informação digital para que, a partir delas, seja possível traduzi-la do formato original para um atual. (FERREIRA, C., 2011)

Um exemplo de aplicação desta estratégia consiste em imprimir em papel um conjunto representativo de documentos de texto juntamente com a sua representação binária. No futuro, as regras necessárias interpretar e migrar os objetos para um novo formato poderiam ser inferidas, comparando os documentos impressos com a sua representação binária. Esta estratégia deverá ser considerada apenas em situações em que todos os esforços de preservação falharam. (FERREIRA, M., 2006, p. 45)

Autores como Heminger e Robertson (1998 apud FERREIRA, C., 2011) defendem a utilização dessa estratégia como alternativa à emulação. Além disso, acreditam que a criação de uma Pedra "que possa ser utilizada para recriar os documentos digitais é uma forma de descrever e preservar o comportamento dos sistemas de informação para a posteridade". (FERREIRA, C., 2011, p. 63)

\subsection{ARQUEOLOGIA Digital}

Por fim, Baggio e Flores (2012) comentam que a arqueologia digital é o resgate das informações digitais contidas em suportes ou formatos já obsoletos e/ou degradados.

Reflete-se em uma solução que apresenta desvantagens visto que, nesta estratégia, pode-se perder conteúdo informacional e, dependendo da quantidade de informação perdida, pode deixar o documento ininteligível ou fazer com que o profissional acabe preenchendo as lacunas com informações que não correspondem ao original, alterando a integridade e autenticidade do documento digital.

De acordo com Carla Ferreira (2011), por conta do seu alto custo e por requerer uma mão-de-obra altamente qualificada, a utilização deste recurso é sugerida apenas para documentos de alta importância, que justifiquem o gasto.

\section{CONCLUSÕES}

Este trabalho teve como objetivo a apresentação de uma revisão de 
literatura que abordasse os desafios da preservação digital diante da obsolescência tecnológica e a fragilidade dos suportes.

O documento digital, criado a partir de uma demanda da sociedade contemporânea por tecnologias da informação, possui complexidades que tangem tanto seu tratamento quanto a sua preservação e o profissional responsável por ele precisa de capacitação específica.

É importante que as organizações reconheçam que o impacto da obsolescência tecnológica é inevitável, uma vez que seu célere avanço é uma realidade da sociedade contemporânea. Ainda que a preservação digital não possa solucionar esse problema, ela deve minimizar as consequências das mudanças nos documentos digitais, evitando a perda da informação.

Deve-se estimular a elaboração de estratégias e políticas para os sistemas de informação, a fim de monitorar mudanças formatos e versões e definir ações em longo prazo.

Este é um assunto que permanece em debate e que não tem uma ação exclusiva para todos os documentos digitais, portanto não há uma resposta simples para o que fazer para solucionar o problema da preservação de documentos digitais com tantas mudanças na tecnologia de produção, tratamento e acesso.

Variados autores destacam como ações para a preservação digital a migração, emulação, encapsulamento e refrescamento junto a um plano que inclua estratégias para a preservação dos componentes físicos, lógicos e conceituais dos documentos digitais.

Portanto, os profissionais de preservação tem uma missão muito complexa e importante, a de manter as informações dos documentos digitais com sua função probatória, autenticidade e integridade com condição de acesso em uma sociedade na qual o avanço e as mudanças nas tecnologias de informação são inevitáveis e a cada dia acontecem com maior rapidez.

\section{REFERÊNCIAS}

BAGGIO, Claudia Carmem; FLORES, Daniel. Estratégias, critérios e políticas para preservação de documentos digitais em arquivos. Ciência da 
Informação, v. 41, n. 2/3, 2012, p. 58-71. Disponível em:

http://revista.ibict.br/ciinf/article/viewFile/1336/1515. Acesso em: 20 abr. 2020.

CONSELHO NACIONAL DE ARQUIVOS (Brasil). Carta para a preservação do patrimônio arquivístico digital. Rio de Janeiro: CONARQ, 2005.

FERREIRA, Carla Alexandra Silva. Preservação da informação digital: uma perspectiva orientada para as bibliotecas. Coimbra: Universidade de Coimbra, 2011.

FERREIRA, Miguel. Introdução à preservação digital: conceitos, estratégias e actuais consensos. Guimarães: Escola de Engenharia da Universidade do Minho, 2006.

INTERNATIONAL RESEARCH ON PERMANENT AUTHENTIC RECORDS IN ELECTRONIC SYSTEMS. Diretrizes do preservador: a preservação de documentos arquivísticos digitais: diretrizes para organizações. [S.I.]: [s.n.], [2013]. Disponível em:

http://www.interpares.org/ip2/display_file.cfm?doc=ip2_preserver_guidelines_bo okl et--portuguese.pdf. Acesso em: 15 abr. 2020.

SANTOS, Henrique Machado; FLORES, Daniel (A). As vulnerabilidades dos documentos digitais: obsolescência tecnológica e ausência de políticas de prática de preservação digital. Biblios, n. 59, 2015, p. 45-54. Disponível em: http://biblios.pitt.edu/. Acesso em: 20 abr. 2020.

SANTOS, Henrique Machado; FLORES, Daniel. O documento digital no contexto das funções arquivísticas. Páginas a\&b, s. 3, n. 5, 2016, p. 165-177. Disponível em:

ojs.letras.up.pt/index.php/paginasaeb/article/download/1477/1271. Acesso em: 5 maio 2020.

SANTOS, Henrique Machado; FLORES, Daniel (B). Estratégias de preservação digital para documentos arquivísticos: uma breve reflexão. Cadernos BAD, n. 1, 2015, p. 87-101. Disponível em:

https://www.bad.pt/publicacoes/index.php/cadernos/article/view/1225/pdf_16. Acesso em: 15 maio 2020.

SANTOS, Henrique Machado; FLORES, Daniel. Os impactos da obsolescência tecnológica frente à preservação de documentos digitais. Brazilian Journal of Information Studies: research trends, v. 11, n. 2, 2017, p.28-37. Disponível em: http://www2.marilia.unesp.br/revistas/index.php/bjis/article/view/5550.

Acesso em: 20 abr. 2020.

SANTOS, Henrique Machado; FLORES, Daniel (C). Políticas de preservação digital para documentos arquivísticos. Perspectivas em Ciência da

Informação, v. 20, n, 4, 2015, p. 197-217.Disponível em:

http://www.scielo.br/pdf/pci/v20n4/1413-9936-pci-20-04-00197.pdf. Acesso em:

5 maio 2020. 
SANTOS, Henrique Machado; FLORES, Daniel (D). Preservação de documentos arquivísticos digitais: reflexões sobre as estratégias de emulação. Encontros Bibli: revista eletrônica de biblioteconomia e ciência da informação. v. 20 , n. 43,2015 , p. 3-19.

THIBODEAU, Kenneth - Overview of technological approaches to digital preservation and challenges in coming years. Council on Library and Information Resources. Pub. 107, 2002. Disponível em: https://www.clir.org/pubs/reports/pub107/thibodeau.html. Acesso em: 9 abr. 2020.

\title{
TECHNOLOGICAL OBSOLESCENCE AND THE FRAGILITY OF DIGITAL DOCUMENT SUPPORTS
}

\begin{abstract}
Introduction: This paper discusses the technological obsolescence and fragility of digital archival documents supports and what implies in their preservation. Objective: A literature review was carried out to analyze the degree to which the discussion on digital preservation is and present proposals for the preservation of digital archival documents in view of the speed of technological advance. Methodology: A bibliographic survey was carried out on the topic published in books, theses, websites and periodical articles, among other materials, mainly in Portuguese. Data collection has a qualitative nature. Results: As a result, it can be considered that technological obsolescence is a consequence of the speed of changes and advances in the area that affect not only the internet but also file formats, software, hardware and operating systems. This forces the preservation professional to act quickly in order to protect the history of the society present in the documents and placed at risk. Conclusions: It is concluded that it is essential to create strategies for digital preservation, with this, it will be possible monitor, plan and treat in long term and minimize the consequences of this obsolescence in order to preserve its originality and authenticity.
\end{abstract}

Descriptors: Technological obsolescence . Digital preservation . Archival documents . Digital documents .

\section{OBSOLESCENCIA TECNOLÓGICA Y FRAGILIDAD DE SOPORTES DE DOCUMENTOS DIGITALES}

\begin{abstract}
RESUMEN
Introducción: Este artículo discute la obsolescencia tecnológica y la fragilidad de los soportes de documentos de archivo digital y lo que esto implica en su preservación. Objetivo: Se llevó a cabo una revisión de la literatura para analizar el grado de discusión sobre la preservación digital y presentar propuestas para la preservación de los documentos de archivo digital en vista de la velocidad del avance tecnológico. Metodología: se realizó una encuesta bibliográfica sobre el tema publicado en libros, tesis, sitios web y artículos periódicos, entre otros materiales, principalmente en portugués. La recopilación de datos tiene una naturaleza cualitativa. Resultados: Como
\end{abstract}


resultado, se puede considerar que la obsolescencia tecnológica es una consecuencia de la velocidad de los cambios y avances en el área que afectan no solo a Internet sino también a los formatos de archivo, software, hardware y sistemas operativos. Esto obliga al profesional de la conservación a actuar rápidamente para proteger la historia de la sociedad presente en los documentos y en riesgo. Conclusiones: se concluye que es esencial crear estrategias para la preservación digital, con esto será posible monitorear, planificar y tratar a largo plazo para minimizar las consecuencias de esta obsolescencia a fin de preservar su originalidad y autenticidad.

Descriptores: Obsolescencia tecnológica .Conservación digital .Documentos de archivo. Documentos digitales .

Recebido em: 27.02.2020

Aceito em: 18.11 .2020 